Article

\title{
Wheat Yellow Mosaic Virus NIb Interacting with Host Light Induced Protein (LIP) Facilitates Its Infection through Perturbing the Abscisic Acid Pathway in Wheat
}

\author{
Tianye Zhang ${ }^{1,2} \oplus$, Peng Liu ${ }^{2}$, Kaili Zhong ${ }^{2}$, Fan Zhang ${ }^{2}$, Miaoze $\mathrm{Xu}^{2}$, Long $\mathrm{He}^{2}$, Peng Jin ${ }^{2}$, \\ Jianping Chen ${ }^{1,2, *}$ and Jian Yang ${ }^{2, *}$ \\ 1 School of Forestry and Biotechnology, Zhejiang Agriculture and Forestry University, Hangzhou 310021, \\ China; ZTye1995@163.com \\ 2 State Key Laboratory for Quality and Safety of Agro-products, Institute of Plant Virology, Ningbo University, \\ Ningbo 315211, China; wood319@126.com (P.L.); zhongkaili@nbu.edu.cn (K.Z.); zf950418@163.com (F.Z.); \\ xumiaoze@yeah.net (M.X.); hnndhelong2@163.com (L.H.); PengJ0310@163.com (P.J.) \\ * Correspondence: jpchen2001@126.com (J.C.); nather2008@163.com (J.Y.); \\ Tel.: +86-571-86404003 (J.C.); +86-571-86419012 (J.Y.)
}

Received: 5 September 2019; Accepted: 22 October 2019; Published: 23 October 2019

\begin{abstract}
Positive-sense RNA viruses have a small genome with very limited coding capacity and are highly reliant on host factors to fulfill their infection. However, few host factors have been identified to participate in wheat yellow mosaic virus (WYMV) infection. Here, we demonstrate that wheat (Triticum aestivum) light-induced protein (TaLIP) interacts with the WYMV nuclear inclusion $\mathrm{b}$ protein $(\mathrm{NIb})$. A bimolecular fluorescence complementation (BIFC) assay displayed that the subcellular distribution patterns of TaLIP were altered by NIb in Nicotiana benthamiana. Transcription of TaLIP was significantly decreased by WYMV infection and TaLIP-silencing wheat plants displayed more susceptibility to WYMV in comparison with the control plants, suggesting that knockdown of TaLIP impaired host resistance. Moreover, the transcription level of TaLIP was induced by exogenous abscisic acid (ABA) stimuli in wheat, while knockdown of TaLIP significantly repressed the expression of ABA-related genes such as wheat abscisic acid insensitive 5 (TaABI5), abscisic acid insensitive 8 (TaABI8), pyrabatin resistance 1-Llike (TaPYL1), and pyrabatin resistance 3-Llike (TaPYL3). Collectively, our results suggest that the interaction of NIb with TaLIP facilitated the virus infection possibly by disturbing the ABA signaling pathway in wheat.
\end{abstract}

Keywords: wheat yellow mosaic virus; BIFC; ABA signaling pathway

\section{Introduction}

Plant RNA viruses are an important cause of agricultural economic losses [1]. It is particularly important to understand the interaction of the virus with plant host factors in the process of infecting plants. Due to their limited coding capacity, RNA viruses recruit some host proteins via direct or indirect interactions to complete their own reproduction and, hence, only survive in living cells [2-4]. More and more evidence supports the idea that versatile host factors are involved in different periods of virus infection. For example, the Potyvirus VPg protein may selectively interact with eukaryotic initiation factor $4 \mathrm{E}$ (eIF4E) or its isoform to regulate virion disassembly via potyviral genome translation, Brome mosaic virus (BMV) 1a can interact with reticulon homology proteins (RHPs) to re-localize them from peripheral ER tubules to the interior of the spherules, and glyceraldehyde-3-phosphate dehydrogenase (GAPDH), a crucial host factor, performs an uncanonical function in TBSV replication 
via interacting with p92 protein [5-7]. Therefore, identifying these host factors at the molecular level and understanding their functions in the process of viral infection could help to develop new disease resistance strategies and reveal novel plant antiviral mechanisms [3].

Potyviruses include many agriculturally and economically important pathogens such as Wheat yellow mosaic virus (WYMV), which belongs to the genus Bymovirus (Potyviridae) [8,9]. The WYMV genome consists of two positive single RNA strands, RNA1 (7.5 kb) and RNA2 (3.6 kb), which each encode a polyprotein [10]. The polyprotein encoded by RNA1 produces eight proteins including the coat protein $(\mathrm{CP})$ and a nuclear inclusion $\mathrm{b}(\mathrm{NIb})$ protein that functions as an RNA-dependent RNA polymerase (RdRp), which is important for virus replication. RNA2 encodes a polyprotein (101 kDa) that gives rise to two proteins of $28 \mathrm{kDa}$ (P1) and $73 \mathrm{kDa}(\mathrm{P} 2)$ [10].

The potyviral $\mathrm{NIb}$ is a nuclear targeting protein and is a vital part of the virus infection process. For example, the (Turnip mosaic virus) TuMV NIb protein interacts with SCE1 and the interaction is necessary for virus infection [11]. Furthermore, SUMOylation of TuMV NIb promotes virus infection by counteracting the NPR1-mediated resistance pathway [12]. NIb is present in viral replication complexes and is capable of recruiting host proteins such as HSP70, NbSCE1, AtRH8, and AtRH9 via interactions to promote potyviral RNA replication [11,13-15]. Furthermore, NIb from several other potyviruses can be recognized by dominant resistance genes (R genes) such as Pvr1, Pvr2, Pvr4, Pvr8, and Pvr9, which encode resistance proteins and trigger a Pvr-mediated hypersensitive response [4,16-20]. Nevertheless, WYMV NIb is not only one of the most conserved portions in the WYMV genome, but is also a good candidate for broad spectrum resistance [21]. Indeed, transgenic wheat containing the antisense virus NIb has durable field resistance to WYMV [21]. In addition, WYMV P1 proteins can interact with NIb and recruit it into P2-induced aggregates through its association with P1 [22]. However, we still do not fully understand the function of WYMV-NIb during the process of viral infection.

To investigate the potential roles of NIb during WYMV infection, we used NIb as a bait to screen a wheat (Triticum aestivum) yeast cDNA library. We obtained light-induced protein (TaLIP) (accession number: AK454210.1), which is a member of the Fibrillin family in wheat, and verified the interaction between TaLIP and NIb in vivo. A bimolecular fluorescence complementation (BiFC) assay indicated that the interaction between $\mathrm{TaLIP}$ and $\mathrm{NIb}$ affects their sub-cellular distribution. Furthermore, the transcription level of TaLIP was downregulated in WYMV-infected wheat and the TaLIP gene silenced wheats were more susceptible to WYMV in comparison to the control wheat plant. Quantitative real-time PCR (qRT-PCR) showed that TaLIP is responsive to external ABA stimuli and silencing the TaLIP downregulated the transcription level of ABA signaling genes such as TaNCED, TaNCED2, TaABI5, TaABI8, TaPYL1, TaPYL3, and TaPYL5. Our results provide a basis for exploring the molecular mechanisms of WYMV pathogenicity in wheat and for providing candidate genes to develop plant transgenic disease resistance.

\section{Materials and Methods}

\subsection{Plant Materials and Plasmids}

Nicotiana benthamiana plants were grown in a glasshouse at $23{ }^{\circ} \mathrm{C}$ with a $16 \mathrm{~h} \mathrm{light} / 8 \mathrm{~h}$ dark photoperiod. WYMV-infected seedlings of Yangmai 158 with typical mosaic symptoms were collected from a diseased nursery in Yantai City, Shandong Province, China.

\subsection{Phylogenetic Analysis and Promoter Cis-Acting Element Prediction Analysis}

Using TaLIP gene and the representative fibrillin genes mainly from Arabidopsis thaliana, Oryza sativa, Triticum aestivum, and several other plants to construct the evolutionary tree. Then, we classified them using a previously reported method of Arabidopsis thaliana fibrillin proteins [23]. The construction of the evolutionary tree utilizes MEGA 4.0 software [24]. 
For the promoter prediction analysis, we obtained the sequence of about $2000 \mathrm{bp}$ before the translation initiation site of TaLIP from the wheat genome database (NCBI), then input this sequence into the PlantCARE database [25] for the promoter prediction analysis.

\subsection{Yeast Two-Hybrid Assay}

Yeast two-hybrid assays were performed following the method described in the Takara protocol handbook. The full length of TaLIP (accession number: AK454210.1) and WYMV NIb were cloned and fused to the Gal DNA-binding domain (vector: pGBKT7) or Gal4 activation domain (vector: pGADT7), respectively, using primers listed in Supplementary Table S2. Yeast cells (strain Y2H Gold) carrying the co-transformed plasmids were plated onto a low-stringency selective medium lacking tryptophan and leucine (SD/-Trp-Leu) to confirm the transformation and plated onto a high-stringency selective medium lacking tryptophan, leucine, histidine, and adenine (SD/-Trp-Leu-His-Ade) to analyze the interaction.

\subsection{Sub-Cellular Localization, Bimolecular Fluorescence Complementation and Co-Immunoprecipitation (Co-IP) Assays}

For subcellular localization analyses and BiFC, a series of recombinant plasmids including NIb-GFP, TaLIP-GFP, NIb-nYFP, and TaLIP-cYFP were constructed using Gateway technology according to the manufacturer's instructions (Invitrogen). The first PCR used the primer pairs NIb-GFPN/NIb-GFPC (NIb for localization), TaLIP-GFPN/TaLIP-GFPC (TaLIP for localization), NIb-nYFPN/NIb-nYFPC (NIb for BiFC), and TaLIP-cYFPN/TaLIP-cYFPC (TaLIP for BiFC) (Supplementary Table S2). The second PCR was performed using primers attB1 and attB2 (Supplementary Table S2) and the amplified product of the first PCR as a template. The amplified product was then introduced into pDONR207 by the BP reaction and the entry clones pENTR-NIb-GFP, pENTR-TaLIP-GFP, pENTR-NIb-nYFP, pENTR-TaLIP-cYFP, and pENTR-TaLIP-HA were constructed. Finally, the LR-clonase reaction was used to transfer NIb and TaLIP fragments from the entry clones to the destination vector and recombinant plasmids including pGWB5C-NIb, pGWB5C-TaLIP, pGTQL1221-NIb, pGTQL1211-TaLIP, and TaLIP-HA were constructed. These recombinants were used to transform the competent E.coli strain DH5 $\alpha$ using heat shock and selected on a medium containing $50 \mu \mathrm{g} / \mathrm{mL}$ kanamycin and $50 \mu \mathrm{g} / \mathrm{mL}$ hygromycin.

The recombinant binary constructs were introduced into Agrobacterium tumefaciens strain GV3101 by electroporation (Bio-Rad Gene Pulser, $0.2 \mathrm{~cm}$ cuvettes, 25 micro F, $>2.1 \mathrm{kV}$ ). Agroinfiltration was performed as described by [4]. Briefly, cultures of GV3101 containing a relevant binary plasmid were grown in yeast extract tryptone (YEP) medium containing rifampicin $(50 \mu \mathrm{g} / \mathrm{mL})$ and kanamycin $(100 \mu \mathrm{g} / \mathrm{mL})$ at $28^{\circ} \mathrm{C}$ for $16 \mathrm{~h}$.

For sub-cellular localization, Agrobacterium cultures containing pGWB5C-NIB and pGWB5C-TaLIP were centrifuged for $30 \mathrm{~s}$ at $8000 \mathrm{rpm}$, resuspended, and then diluted to an $\mathrm{OD}_{600}$ of 0.6 (10 mM MES, $\mathrm{pH} 5.6,10 \mathrm{mM} \mathrm{MgCl} 2,200 \mathrm{mM}$ acetosyringone) before leaf infiltration. The expression of fluorescent proteins was examined at $72 \mathrm{~h}$ post agroinfiltration.

For the BiFC assay, GV3101 strains containing the BiFC plasmids with pGTQL1221-NIb-nYFP and pGTQL1211-TaLIP-cYFP were resuspended and adjusted to an $\mathrm{OD}_{600}$ in a 1:1 ratio with infiltration medium before leaf infiltration. The combinations pGTQL1221-NIb-nYFP/pGTQL1211-GUS-cYFP and pGTQL1221-GUS-nYFP/pGTQL1211-TaLIP-cYFP were used as negative controls. The cell suspensions were incubated at room temperature for $2 \mathrm{~h}$ to $4 \mathrm{~h}$ and then used to infiltrate 5- to 6-week-old $N$. benthamiana leaves. The expression of fluorescent proteins was examined at $72 \mathrm{~h}$ post agroinfiltration [26]. 
For the in vivo co-IP analysis, about $0.5 \mathrm{~g}$ Agro-infiltrated leaf tissue frozen in liquid nitrogen was ground to a fine powder and thawed in plant protein extraction buffer containing $10 \%$ glycerol, $25 \mathrm{mM}$ Tris- $\mathrm{HCl}$, pH 7.5, $1 \mathrm{mM}$ EDTA, $150 \mathrm{mM} \mathrm{NaCl}$, 2\% polyvinylpolypyrrolidone (PVPP), $10 \mathrm{mM}$ DTT, $1 \times$ protease inhibitor cocktail (Sigma, Shanghai, China), 0.2\% Triton X-100 (Sigma-Aldrich, St. Louis, MO, USA) ( $1 \mathrm{~g}$ tissue per sample/2 $\mathrm{mL}$ buffer). The mixture was centrifuged at $18,000 \mathrm{~g}$ for $10 \mathrm{~min}$ at $4{ }^{\circ} \mathrm{C}$. Each supernatant $(500 \mu \mathrm{L})$ was mixed with $45 \mu \mathrm{L}$ anti-GFP conjugated agarose beads (Sigma) and incubated at $4{ }^{\circ} \mathrm{C}$ for $1.5 \mathrm{~h}$ with gentle shaking. Agarose beads were pelleted and washed three times with the co-IP buffer (10\% glycerol, $25 \mathrm{mM}$ Tris-HCl, pH 7.5, $1 \mathrm{mM}$ EDTA, $150 \mathrm{mM} \mathrm{NaCl}, 2 \%$ PVPP, $1 \mathrm{mM}$ DTT, $0.1 \%$ Triton X-100). The resulting pellets were mixed individually with SDS loading buffer boiled at $100{ }^{\circ} \mathrm{C}$ for $8 \mathrm{~min}$. For immunoblot, proteins were separated in $10 \%$ SDS-PAGE gels through electrophoresis, and then transferred to NC membranes. The blots were probed with an anti-HA (1:5000), anti-GFP (1:5000), followed by an HRP-conjugated secondary antibody. The detection signals were developed using an electrochemiluminescence (ECL) reagent as instructed (Thermo Scientific, Hudson, NH, USA), and visualized using a Bio-Rad ChemiDoc Touch imaging system (Bio-Rad, Hercules, CA, USA).

\subsection{Plant RNA Isolation and Quantitative Real Time PCR Analysis}

Leaves were collected from infected wheat plants, frozen, and stored at $-80^{\circ} \mathrm{C}$ until use. Total RNAs were extracted from plants using Trizol reagent (Invitrogen) and stored at $-80^{\circ} \mathrm{C}$. Quantitative real time (qRT)-PCR analysis was performed using an ABI7900HT Sequence Detection System (Applied Biosystems, CA, USA) with an AceQ qPCR SYBR Green Master Mix (Vazyme, Nanjing, Jiangsu, China). At least three biological replicates, with three technical replicates, were used for each assay. The Triticum aestivum cell division cycle (CDC) gene (Accession Number: XM_020313450) was used as the internal reference gene for analysis to calculate the fold changes in gene expression. The fold changes were calculated using the $2^{-\Delta \Delta C(t)}$ method [27]. All gene-specific primers for qRT-PCR are shown in Supplementary Table S2.

\subsection{Virus-Induced Gene Silencing}

Barley stripe mosaic virus (BSMV)-based gene silencing vectors were kindly provided by Dr Dawei Li, China, and have been widely used in barley and wheat [28]. TaLIP (200 bp) from the leaf cDNA of wheat was amplified by RT-PCR (Supplementary Table S2), inserted into the BSMV $\gamma$ gene (BSMV:TaLIP), and digested with Pac I and Not I restriction enzymes. Additionally, the BSMV:00 was used as the negative control.

\subsection{Mechanical Friction Inoculation of Barley stripe mosaic virus and Wheat yellow mosaic virus}

Virus in vitro transcription followed by friction inoculation was performed as previously described [29,30]. Briefly, in vitro transcription of linearized plasmid transcripts of BSMV RNA $\alpha, \beta$, and $\gamma$ in a molar ratio of 1:1:1 were mixed with an equal amount of excess inoculation buffer (named as FES) (0.06 M potassium phosphate, $0.1 \mathrm{M}$ glycine, $1 \%$ bentonite, $1 \%$ sodium pyrophosphate decahydrate, $1 \%$ celite, $\mathrm{pH} 8.5$ ) and then inoculated into leaves of 7-10-day-old wheat seedlings. In vitro transcription of linearized plasmid transcripts of WYMV RNA R1 and R2 were also mixed at a molar ratio of 1:1, then inoculated into the upper leave of the BSMV-infected wheat seedling.

\section{Results}

3.1. TaLIP Interacts with Wheat Yellow Mosaic Virus NIb and C-Terminus of NIb ${ }^{196-380 a a}$ Is the Major Region for This Interaction

$\mathrm{NIb}$ plays an important role in the process of virus infection. To investigate the host factors that interact with the $\mathrm{NIb}$, we used $\mathrm{NIb}$ as a bait to screen a wheat (Triticum aestivum) yeast cDNA library. A series of proteins were identified via the yeast two-hybrid screening technology including two 
clones of partial fragment of chlorophyll a-b binding protein 50, eight clones of partial fragment of light-induced protein, and five clones of full length of GDP-L-galactose phosphorylase (Supplementary Table S1). Consider the number of selected proteins, the light-induced protein was selected for further investigation. The sequence alignment between Triticum aestivum light-induced protein and its homologous genes of Nicotiana tabacum, Capsicum baccatum, Helianthus annuus, and Lactuca sativa indicated that the homology was $83.23 \%$ (Supplementary Figure S1). For convenience, we named Triticum aestivum light-induced protein as TaLIP in this study. Subsequently, yeast two-hybrid assays also demonstrated an interaction between TaLIP and WYMV NIb (Figure 1a). Furthermore, to determine the key domain for interaction, WYMV NIb (380 aa) was further divided into two smaller fragments, $\mathrm{NIb}^{1-195}$ (encoding aa 1-195) and $\mathrm{NIb}^{196-380}$ (encoding aa 196-380) for yeast two-hybrid assays (Figure 1b). Although both $\mathrm{NIb}^{1-195}$ and $\mathrm{NIb}^{196-380}$ interacted with TaLIP, the yeast two-hybrid assays revealed a stronger interaction between $\mathrm{NIb}^{196-380}$ and TaLIP (Figure 1c). Thus, residues 196 to 380 of NIb appear to be crucial for its interaction with TaLIP.
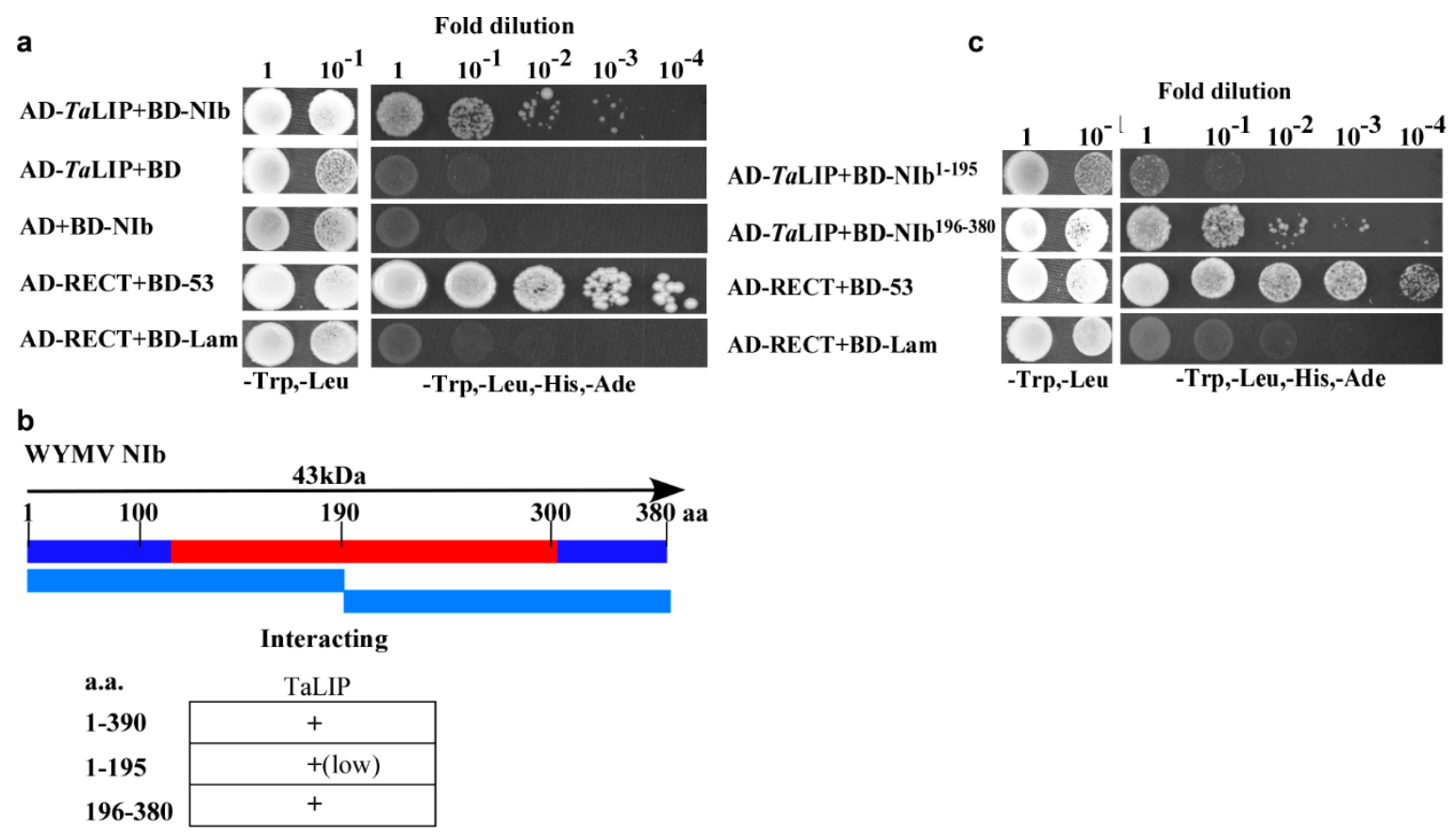

Figure 1. Interaction of Wheat yellow mosaic virus (WYMV)NIb with and TaLIP in a yeast two-hybrid analysis. (a) NIb was fused to the DNA-binding domain and TaLIP was fused to the activation domain (AD-TaLIP), co-transformed into yeast cells, and then coated uniformly on selection plates of SD/-Trp, -Leu, -Ade, -His solid medium. Positive and negative controls were co-transformed with AD-T/BK-53 and AD-T/BK-Lam, respectively. (b) NIb was divided into two parts and fused to the DNA-binding domain, co-transformed with AD-TaLIP into yeast cells, and then coated uniformly on selection plates of SD/-Trp, -Leu, -Ade, and -His solid medium. AD-T/BK-53 and AD-T/BK-lam were used as the positive control and negative control, respectively. (c) Illustration of the WYMV NIb sequence, which divided into two segments and summary of TaLIP interactions with WYMV NIb. The red region represents the conserved domains of NIb.

\subsection{TaLIP Interacts with WYMV NIb In Vivo}

To test the interaction between NIb and TaLIP in vivo, co-immunoprecipitation (Co-IP) assays were conducted. For the Co-IP assays, GFP-tagged NIb (NIb-GFP) was transiently co-expressed with HA-tagged TaLIP (TaLIP-HA) and GFP-tagged was transiently co-expressed with TaLIP-HA in $N$. benthamiana leaves as the control. Leaf tissues were then collected at $60 \mathrm{~h}$ post infiltration (hpi). Total protein extracts were immunoprecipitated using anti-GFP antibody coupled to agarose beads, and the resulting precipitates were analyzed by immunoblot using anti-HA antibodies. We observed 
that TaLIP co-immunoprecipitated with NIb-GFP, but not with GFP alone (Figure 2). Taken together, these results further demonstrate an interaction between WYMV NIb and TaLIP in vivo.

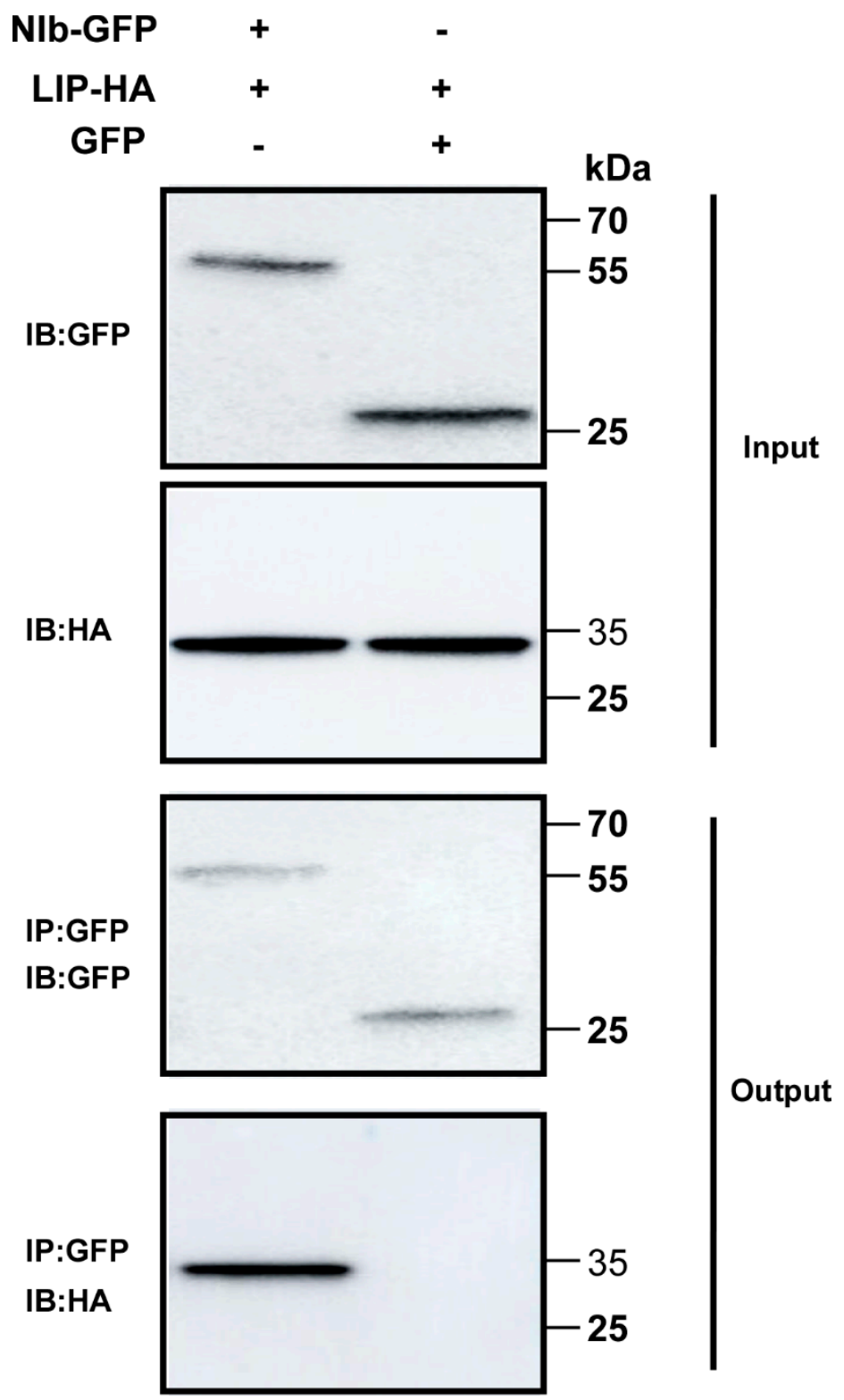

Figure 2. Co-immunoprecipitation analysis of the interaction between $\mathrm{NIb}$ and $\mathrm{TaLIP}$. The Green fluorescent protein (GFP) was fused at the C-terminus of NIb (NIb-GFP) and transiently co-expressed with the TaLIP-HA in N. benthamiana leaves. GFP-tagged was transiently co-expressed with TaLIP-HA in N. benthamiana leaves as the control. The TaLIP-His tag (HA) was used to co-immunoprecipitate with NIb-GFP. The blots were probed with a GFP specific antibody or a HA specific antibody. IP, immunoprecipitation with special antibody. IB, immunoblot with special antibody. The sizes of the proteins in $\mathrm{kDa}$ are shown to the left. 


\subsection{Sub-Cellular Localization of WYMV NIb and TaLIP Is Altered by Their Interaction in} Nicotiana Benthamiana

To determine whether the sub-cellular localization of $\mathrm{TaLIP}$ and $\mathrm{NIb}$ was affected by the interaction of these proteins, the recombinant plasmids expressing TaLIP or WYMV NIb fused with Green fluorescent protein (GFP) at their C terminus (TaLIP:GFP and NIb:GFP) were constructed and introduced into N. benthamiana epidermal cells by Agrobacterium infiltration. At $72 \mathrm{~h}$ post infiltration (hpi), GFP fluorescence was detected by confocal microscopy. TaLIP:GFP was observed in the chloroplast and $\mathrm{NIb}$ :GFP was observed in the nucleus and cytoplasm (Figure 3). Subsequently, we performed BiFC assays to analyze the interactions between TaLIP and WYMV NIb. pGTQL1211-GUS-cYFP and pGTQL1221-GUS-nYFP were used as the two negative controls, respectively. These combinations of fusion proteins were all expressed in N. benthamiana via agroinfiltration. When pGTQL1221-NIb-nYFP (YN-NIb) and pGTQL1211-TaLIP-cYFP (YC-TaLIP) were co-expressed in N. benthamiana epidermal cells, some aggregates were observed in the cytoplasm and strong GFP signals (we changed the pseudo-color of the YFP signal to green for convenience) were observed around the chloroplast, but not in the chloroplast. In contrast, no significant fluorescence signal was detected in the negative controls (Figure 4). It is interesting that the sub-cellular localization of TaLIP and WYMV NIb were completely different from that observed when performing the BiFC assay. Based on these data, we speculate that the interaction between NIb and TaLIP may affect the distribution patterns of WYMV NIb and TaLIP.
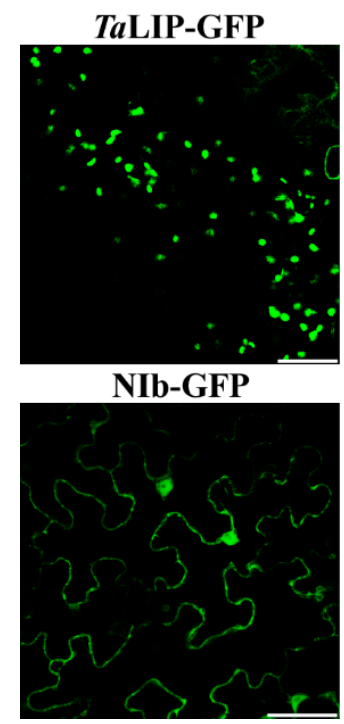

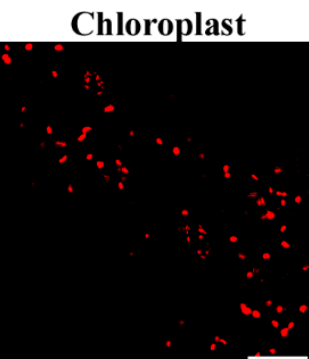

Chloroplast

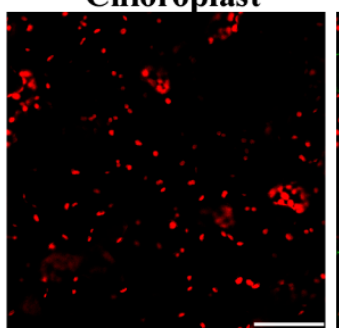

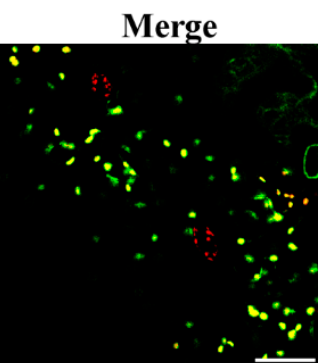

Merge

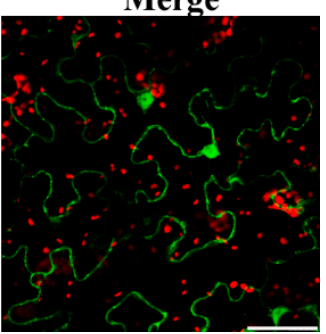

Figure 3. Localization of the TaLIP and WYMV NIb protein in Nicotiana benthamiana leaves agroinfiltrated with pENTR-NIb-GFP or pENTR-TaLIP-GFP. The green fluorescent signal of TaLIP proteins was co-localized with the red auto-fluorescent signal of chloroplasts. Fluorescence photographs were taken at 3 dpi. Scale bar: $50 \mu \mathrm{m}$. 
YN-NIb+YC-TaLIP
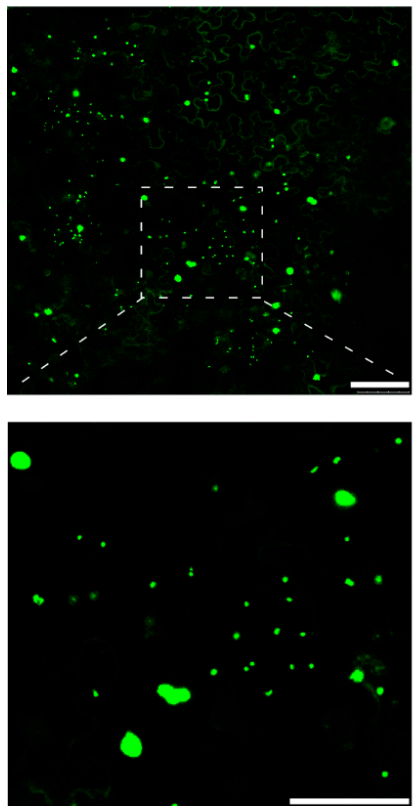

YN-GUS+YC-TaLIP

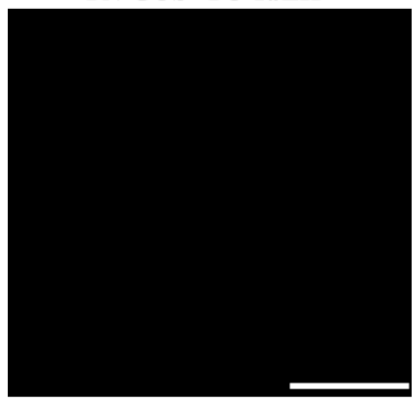

YN-NIb+YC-GUS

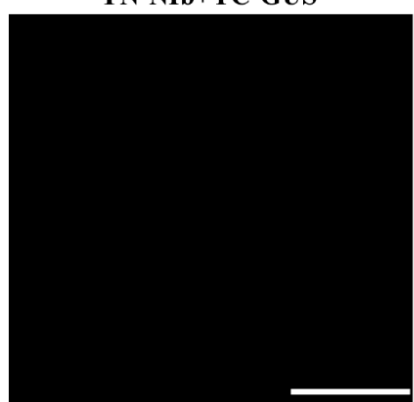

Chloroplast
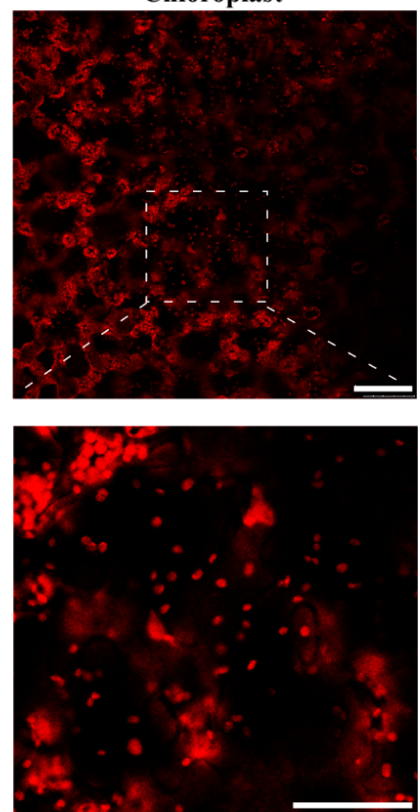

Chloroplast

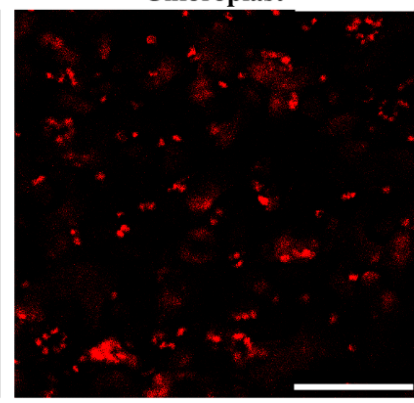

Chloroplast

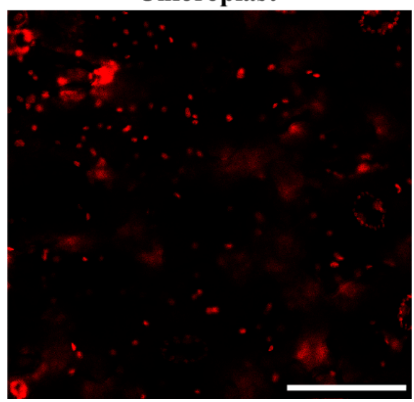

Merge
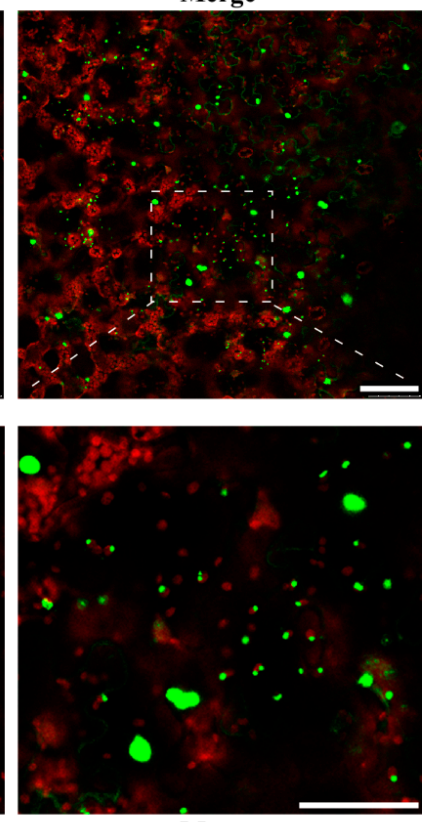

Merge

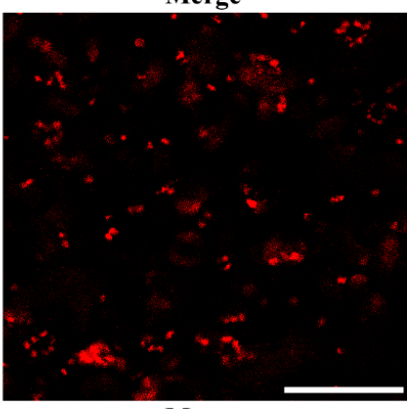

Merge

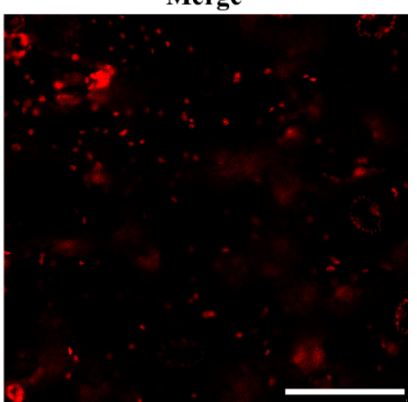

Figure 4. Bimolecular fluorescence complementation (BiFC) assay of the WYMV NIb and TaLIP interactions in Nicotiana benthamiana. The pseudo-color of the Yellow fluorescent protein (YFP) signal was changed into green for convenience. The red fluorescent signal is a chloroplast auto-fluorescence signal. $\beta$-Galactosidase (GUS)-fused vectors pGTQL1211-GUS-cYFP/pGTQL1221-NIb-nYFP (YN-NIb/YC-GUS) and pGTQL1221-GUS-nYFP/pGTQL1211-TaLIP-cYFP (YN-GUS/YC-TaLIP) are the negative controls. Fluorescence photographs were taken at 3 dpi. Scale bar: $50 \mu \mathrm{m}$.

\subsection{The Transcriptional Level of TaLIP Is Downregulated in WYMV-Infected Wheat}

To clarify whether TaLIP was affected at the transcriptional level by WYMV infection, we then designed primer pairs for quantitative RT-PCR (qRT-PCR) and characterized the expression profile of TaLIP in Triticum aestivum with or without WYMV infection. The expression level of TaLIP was significantly downregulated 0.23-fold in WYMV-infected wheat when compared to that of WYMV-uninfected wheat (Figure 5). These results indicate that the expression of TaLIP in the WYMV-infected wheat plants can be regulated, potentially to benefit WYMV infection in this host. 


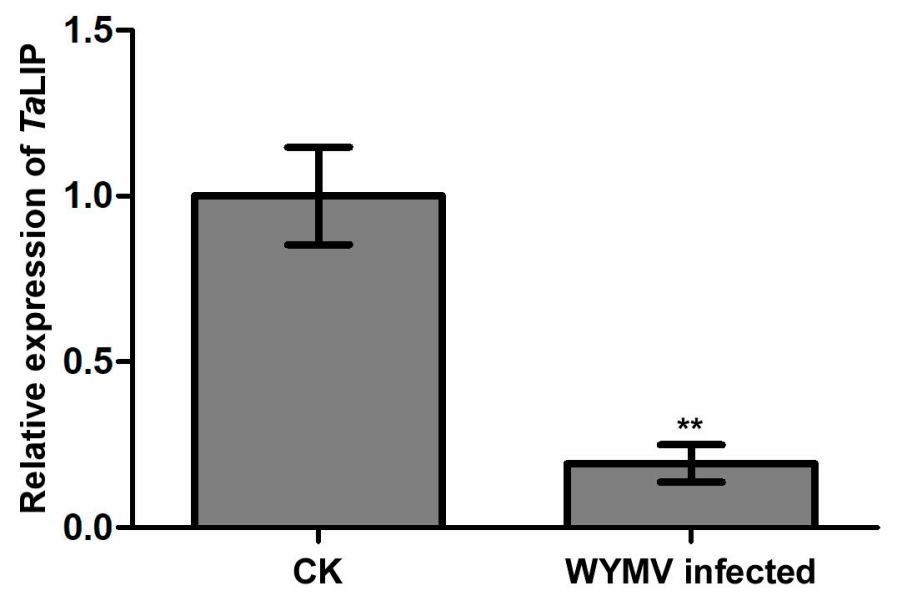

Figure 5. The expression level of TaLIP was significantly downregulated in WYMV-infected wheat. $T a C D C$ was used as the internal control. The level of TaLIP in health wheat was normalized to 1. Each relative expression level is presented as the mean \pm SD from four biological samples and each biological sample had four technical replicates. Statistical analyses were done using the Student's $t$-test. Asterisks indicate a significant difference when compared to the control. ${ }^{*} p<0.05 ;{ }^{* *} p<0.01$.

\subsection{Knockdown the TaLIP Facilitate WYMV Infection in Wheat}

To investigate the relationship between TaLIP expression and WYMV infection in wheat, we inoculated six wheat seedling with an RNA transcript representing BSMV + WYMV or BSMV:TaLIP + WYMV. After $7 \mathrm{dpi}$, we analyzed the silencing level of the TaLIP gene in the BSMV:TaLIP + WYMV co-inoculated wheat seedling through qRT-PCR using TaLIP specific primers. The results showed that the TaLIP transcript level in the plants co-inoculated with BSMV:TaLIP + WYMV were better silenced $(p<0.01)$ than the plants co-inoculated with BSMV + WYMV (Figure 6a). Then, the expression level of WYMV CP was also detected by qRT-PCR using the CP specific primers in these plants. The results showed that the expression level of WYMV CP was detected by qRT-PCR and the WYMV CP expression level of BSMV:TaLIP + WYMV inoculated wheat was significantly higher than the inoculated wheat (BSMV + WYMV) (Figure 6b). These results suggest that knockdown of TaLIP impaired host resistance.

a

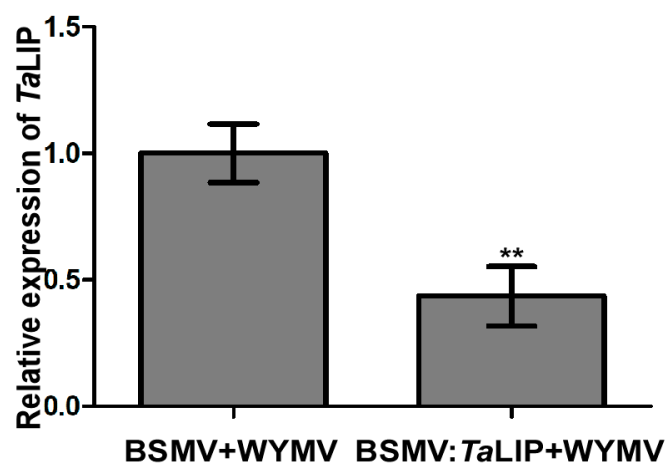

b

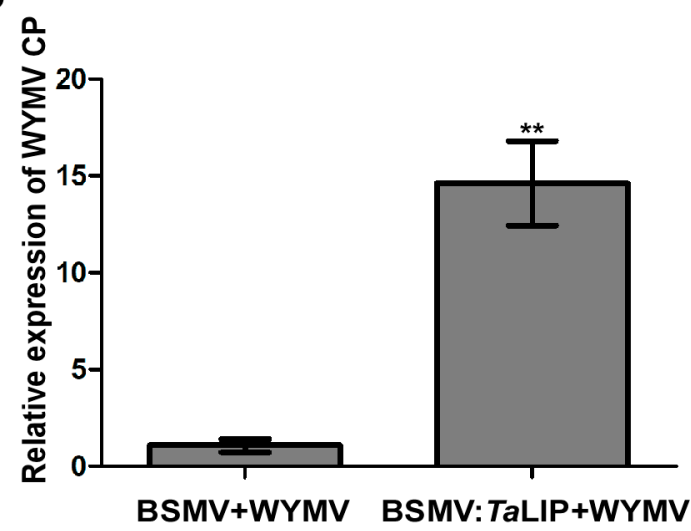

Figure 6. Silencing TaLIP gene expression through virus-induced gene silencing (VIGS) significantly promoted WYMV infection in Triticum aestivum. (a) The quantitative RT-PCR analysis of WYMV $\mathrm{CP}$ expression in the inoculated leaves harvested from the TaLIP-silenced or non-silenced plants. (b) Quantitative RT-PCR analysis of TaLIP expression silenced by BSMV-mediated VIGS in Triticum aestivum. The TaCDC was used as the internal control. Bar represents the SD of three experiments (each with three technical replicates), Asterisks indicate ${ }^{* *} p<0.01$ to the amount of relative WYMV CP and TaLIP expression by the Student's $t$-test. 
3.6. TaLIP Belongs to the FBN1 Subspecies of the Fibrillin Family and Has Three Copies of ABA Responsive Promoter Element

Based on the above findings, we can speculate that NIb interacting with TaLIP might facilitate the WYMV infection by perturbing the pathway mediated by TaLIP in wheat. To predict the potential function of TaLIP in the process of WYMV infection, the phylogenetic relationship of TaLIP between other FBN proteins was constructed. Fourteen Arabidopsis thaliana FBN proteins, eight Oryza sativa FBN proteins, 12 Triticum aestivum FBN proteins, and nine other plant FBN proteins were selected for further analyses. The phylogenetic analysis was performed using the MEGA4 program [24]. Phylogenetic analysis indicated that TaLIP was categorized into subfamily FBN1 (Figure 7a). There is some evidence that the plant hormones regulate FBN gene expression in plants such as ABA [31]. Interestingly, the Cis-acting element information of the TaLIP gene showed that there were nine copies of the ABA responsive promoter element (Figure $7 \mathrm{~b}$ ). We predicted that the TaLIP gene is involved in the response to ABA.

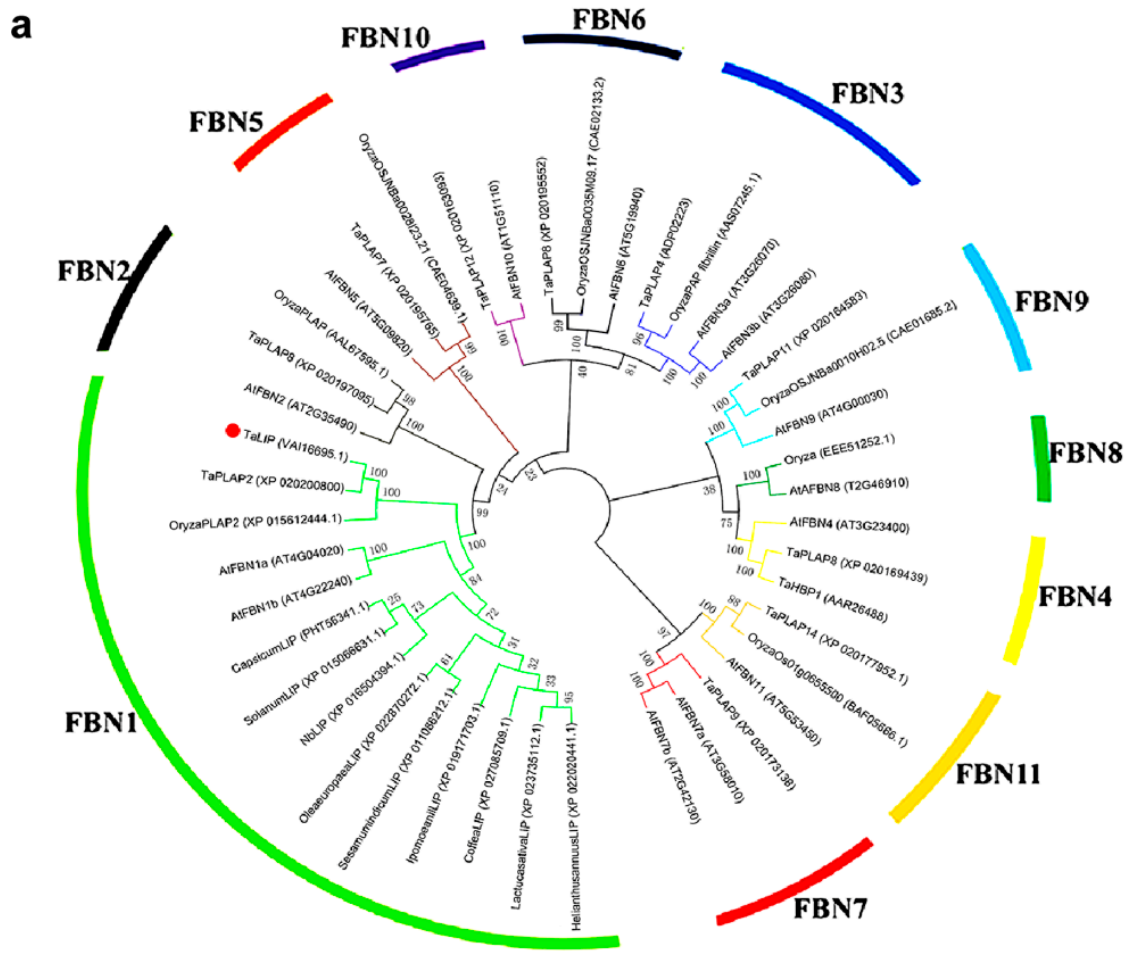

b

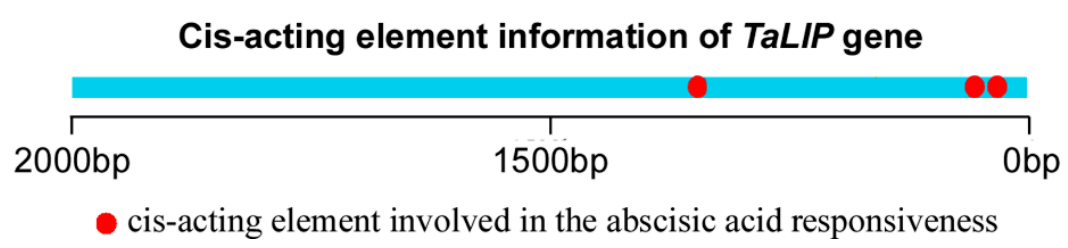

Figure 7. The TaLIP protein belongs to the FBN1 (fibrillin family) and have three copies of the abscisic acid (ABA) responsive element. (a) Phylogenetic analysis of TaLIP and the construction of the evolutionary tree utilizing MEGA4 program [24]. The 11 distinct subfamilies were designated as 1 11 and labeled with different colored branches respectively. (b) promoter Cis-acting element prediction analysis of TaLIP. 
3.7. TaLIP Is Responsive to External ABA Stimuli, Silencing the TaLIP Gene Suppressing the ABA Signaling Pathway

To determine how TaLIP responds to exogenous applications of hormones, we investigated the transcription profiles of TaLIP in wheat treated with ABA. After ABA treatment, expression was induced from 0.5 to $12 \mathrm{~h}$ post-treatment (hpt), rapidly increased to 2.15 -fold at $0.5 \mathrm{hpt}$, reached a peak at $6 \mathrm{hpt}$ (about 3.29-fold that of nontreatment), then decreased to 2.60-fold higher than the nontreated control (N) from 6 to $12 \mathrm{hpt}$ (Figure 8a). These results suggest that TaLIP transcription was induced by exogenous ABA stimuli. Next, we investigated the expression of mRNA level of the ABA-related genes including the ABA biosynthetic pathway gene TaNCED, TaNCED2, and the ABA signaling pathway gene TaABI5, TaABI8, TaPYL1, TaPYL3, and TaPYL5 in TaLIP-silenced wheat (Figure 8b). The transcription level of TaABI5, TaABI8, TaPYL1, and TaPYL3 were suppressed in TaLIP-silenced wheat.

a

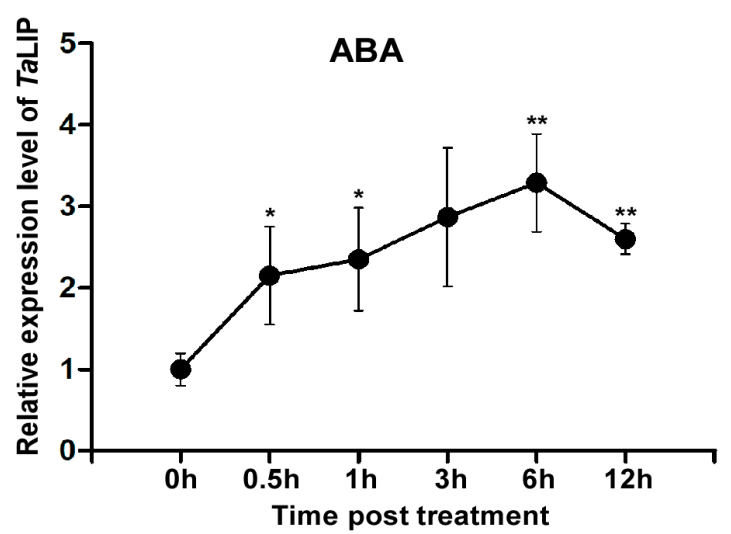

b

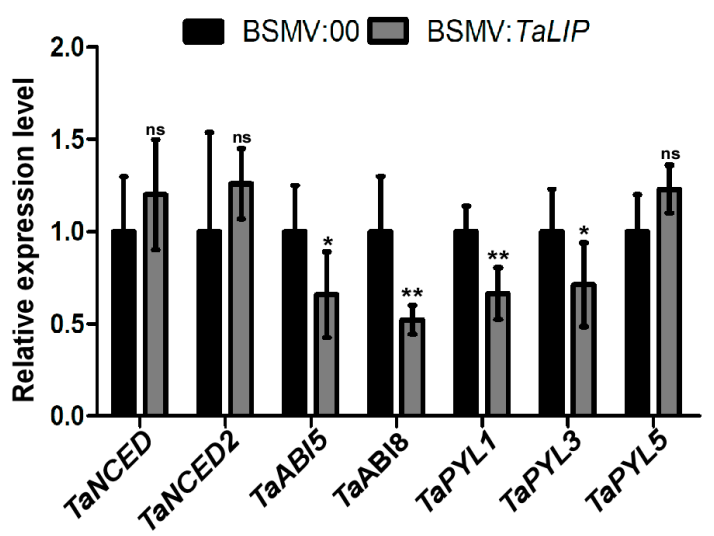

Figure 8. Exogenous ABA treatment induced the expression of TaLIP and the ABA signaling pathway was suppressed in TaLIP-silenced wheat. (a) Expression patterns of TaLIP in wheat after treatment with exogenous hormones ABA for 0.5, 1, 3, 6, and $12 \mathrm{~h}$. Relative expression of TaLIP is shown as fold change in transcription over the non-treatment control $(0 \mathrm{~h})$. Each relative expression level is presented as the mean \pm SD from three biological samples and each biological sample had four technical replicates. Statistical analyses were done using the Student's $t$-test. Asterisks indicate a significant difference when compared to the control. ${ }^{*} p<0.05$; ${ }^{* *} p<0.01$. (b) Quantitative RT-PCR analysis of TaNCED, TaNCED2, TaABI5, TaABI8, TaPYL1, TaPYL3, and TaPYL5 expression in the inoculated leaves harvested from the TaLIP-silenced or non-silenced wheat. The TaCDC was used as the internal control. Each relative expression level is presented as the mean \pm SD from three biological samples and each biological sample had four technical replicates. Statistical analyses were done using the Student's $t$-test. Asterisks indicate a significant difference when compared to the control. ${ }^{*} p<0.05 ;{ }^{* *} p<0.01$.

\section{Discussion}

Numerous studies have demonstrated that to cause an infection in plants, virus have evolved to encode factor(s) to defeat plant defense machinery. As stated in the introduction, the potyviral NIb is a vital protein response for virus infection. However, we still do not fully understand the function of WYMV-NIb during the viral infection. In this study, we obtained a fibrillin (FBN) protein, TaLIP, by performing yeast two-hybrid screening, that could interact with $\mathrm{NIb}$ and the C-terminal of NIb was the key domain for this interaction (Figure 1). Then, the co-IP assay confirmed the interaction between $\mathrm{NIb}$ and TaLIP (Figure 2). FBNs are a large protein family present in photosynthetic organisms ranging from cyanobacteria to higher plants [32], and FBNs are involved in plant responses to biotic stress [23]. Previous study has demonstrated that the $\mathrm{NIb}$ of several potyvirals can interact with host protein(s) to promote viral infections such as HSP70, NbSCE1, and AtRH9 [11,13,15]. Additionally, recent research has shown that fibrillin/CDSP 34 (FBN1) protein levels decreased in tobacco plants infected with tobacco mosaic virus (TMV), which supported our result that the TaLIP transcription level of 
WYMV-infected wheat was significantly downregulated when compared to that of WYMV-uninfected wheat (Figure 5). Therefore, we predicted that TaLIP is possibly involved in the process of WYMV infection. Some researchers have proven that FBNs are involved in disease resistance. For example, knockdown of expression of the LeChrC (FBN1) gene in tomato caused greater susceptibility to Botrytis cinereal; knockdown of FBN4 expression in apple and a mutation of FBN4 in Arabidopsis caused greater susceptibility to the pathogenic bacteria Erwinia amylovora and Pseudomonas syringae pv. tomato, respectively; and a mutant of the FBN1b in Arabidopsis was more susceptible to $P$. syringae pv. Maculicola $[31,33,34]$. Consistent with these findings, knockdown of TaLIP expression facilitated WYMV infection in wheat (Figure 6). According to these data, we suggest that Nib-TaLIP interaction possibly promotes WYMV infection. Many FBNs have been previously reported to be chloroplast related proteins such as cucumber CHRC (FBN1) [35]. Consistently, sub-cellular localization analysis showed that TaLIP was also located in chloroplast (Figure 3). Previous study has demonstrated that the NIb of several other potyvirals was located in the nucleus and cytoplasm [12]. Consistent with this, sub-cellular localization analysis of $\mathrm{NIb}$ showed that $\mathrm{NIb}$ was also located in the nucleus and cytoplasm (Figure 3). Interestingly, BiFC assay revealed a fact that the NIb-TaLIP interaction completely changed their sub-cellular localization in $N$. benthamiana epidermal cells and a large number of fluorescent signals around the chloroplast, but not in the chloroplast (Figure 4). It is possible that Nib-TaLIP interaction affects the function of TaLIP through changing the sub-cellular localization to facilitate WYMV infection. This assumption is supported by findings showing that TuMV NIb interacting with NbEXPA1 recruits NbEXPA1 to the viral replication complex and promotes TuMV infection [9]. Early study has shown that the negatively regulated ABA responses abscisic acid insensitive 2 (ABI2) interacts with the Arabidopsis FNB1a to regulate its localization, leading to perturbing the ABA pathway [36]. In addition, ABA appears to enhance plant antiviral defense as shown for several viruses [37-39]. Hereby, we reasonably speculate the interaction between the NIb and TaLIP facilitated the WYMV infection by affected the ABA pathway associated with TaLIP. Indeed, there is some evidence that hormones can regulate FBN gene expression [23]. For example, in chromoplasts of bell pepper fruit, indole-3-acetic acid can delay the accumulation of FBN protein, whereas ABA can accelerate it [40]. Indeed, the promoter Cis-acting element assay of TaLIP showed that it has three copies of the ABA responsive promoter element (Figure $7 \mathrm{~b}$ ), and the expression of the TaLIP gene was significantly induced by external ABA stimuli (Figure 8a). Thus, we speculate that TaLIP is a responsible gene involved in the ABA signal pathway. Consistent with this notion, the mRNA expression level of ABA pathway genes TaABI5, TaABI8, TaPYL1, and TaPYL3 were downregulated in TaLIP-silenced wheat (Figure 8b). TaABI5 and TaABI8 were reported as positive modulators of ABA signaling and PYL 1 and PYL3 were reported to be $A B A$ receptors positively responsive to $A B A$ [41]. While transcriptome analysis revealed that the transcription levels of TaABI5, TaABI8, TaPYL1, and TaPYL3 were also significant lower in WYMV-infected wheat when compared to that of healthy wheat (Supplementary Figure S2). In the last few decades, ABA has been reported as a key hormone involved in tuning responses to several abiotic stresses and also induces different resistance mechanisms to viruses, regardless of the induction time [33]. Taken together, our work revealed that WYMV NIb interacts with host TaLIP to promote the WYMV infection possibly through affecting the ABA signal pathway.

\section{Conclusions}

In this study, we analyzed the function of the TaLIP gene in the process of WYMV infection. The sub-cellular localization changes of TaLIP and NIb under the interaction indicated that the TaLIP-NIb interaction may affect the function of TaLIP. Furthermore, the result of the TaLIP knockdown experiment indicated that TaLIP may act as a host protein interacting with WYMV NIb to facilitate WYMV infection. External ABA stimuli and the ABA pathway gene expression of TaLIP silenced wheat results indicated that TaLIP may be a responsible gene involved in the ABA signal pathway. In summary, our study revealed that WYMV NIb interacts with host TaLIP to promote the WYMV infection possibly through affecting the ABA signal pathway. Additionally, this work provides a basis 
for exploring the molecular mechanisms of WYMV pathogenicity in wheat and in providing candidate genes to develop plant transgenic disease resistance.

Supplementary Materials: The following are available online at http://www.mdpi.com/2079-7737/8/4/80/s1, Figure S1. Sequence homology alignment between LIP homologous genes of six different species; Figure S2. Transcriptome analysis of WYMV-infected wheat. Healthy wheat was used as the negative control; Table S1. Information of proteins list obtained from Yeast two-hybrid assay; Table S2. The sequences of the primers used in these experiments.

Author Contributions: J.Y. and J.C. conceived the project and designed the experiments; T.Z. and J.Y. carried out the experiments with assistance from P.L., K.Z., F.Z., M.X., L.H., and P.J.; all authors analyzed and discussed the results; and J.Y., T.Z., and J.C. wrote the manuscript.

Funding: This work was supported by the National Key R\&D Plan in China (2018YFD0200408, 2018YFD0200507, 2017YFD-0201701), the China Agriculture Research System from the Ministry of Agriculture of the P.R. China (CARS-03), the National Key Project for Research on Transgenic Biology (2016ZX08002-001), the international science and technology cooperation program of China (2012DFA30900), and the K.C. Wong Magna Funding, Ningbo University.

Acknowledgments: We thank Da-Wei Li (State Key Laboratory of Agro-Biotechnology, China Agricultural University) for providing the BSMV vector.

Conflicts of Interest: The authors have declared that no competing interest exist.

\section{References}

1. Sanfaçon, H.J. Grand challenge in plant virology: Understanding the impact of plant viruses in model plants, in agricultural crops, and in complex ecosystems. Front. Microbiol. 2017, 8, 860. [CrossRef] [PubMed]

2. García, J.A.; Pallás, V.J. Viral factors involved in plant pathogenesis. Curr. Opin. Virol. 2015, 11, 21-30. [CrossRef] [PubMed]

3. Li, Y.; Cui, H.; Cui, X.; Wang, A.J. The altered photosynthetic machinery during compatible virus infection. Curr. Opin. Virol. 2016, 17, 19-24. [CrossRef] [PubMed]

4. Wang, A.J. Dissecting the molecular network of virus-plant interactions: The complex roles of host factors. Annu. Rev. Phytopathol. 2015, 53, 45-66. [CrossRef] [PubMed]

5. Torrance, L.; Andreev, I.A.; Gabrenaite-Verhovskaya, R.; Cowan, G.; Mäkinen, K.; Taliansky, M.E.J. An unusual structure at one end of potato potyvirus particles. J. Mol. Biol. 2006, 357, 1-8. [CrossRef]

6. Arturo, D.; Xiaofeng, W.; Paul, A.J. Membrane-shaping host reticulon proteins play crucial roles in viral RNA replication compartment formation and function. Proc. Natl. Acad. Sci. USA 2010, 107, 16291-16296.

7. Wang, Y.L.; Nagy, P.D.J. Tomato bushy stunt virus Co-Opts the RNA-Binding Function of a Host Metabolic Enzyme for Viral Genomic RNA Synthesis. Cell Host Microbe 2008, 3, 178-187. [CrossRef]

8. Revers, F.; García, J.A. Molecular biology of potyviruses. Adv. Virus Res. 2015, 92, 101-199.

9. Park, S.H.; Li, F.; Renaud, J.; Shen, W.; Li, Y.; Guo, L.; Cui, H.; Sumarah, M.; Wang, A. NbEXPA1, an $\alpha$-expansin, is plasmodesmata-specific and a novel host factor for potyviral infection. Plant J. 2017, 92, 846-861. [CrossRef]

10. Nakamura, K.; Fushimi, K.; Kouchi, H.; Mihara, K.; Miyazaki, M.; Ohe, T.; Namba, M.J. Inhibitory effects of antioxidants on neonatal rat cardiac myocyte hypertrophy induced by tumor necrosis factor- $\alpha$ and angiotensin II. Circulation 1998, 98, 794-799. [CrossRef]

11. Xiong, R.J. SCE1, the SUMO-conjugating enzyme in plants that interacts with NIb, the RNA-dependent RNA polymerase of Turnip mosaic virus, is required for viral infection. J. Virol. 2013, 87, 4704-4715. [CrossRef] [PubMed]

12. Cheng, X.; Xiong, R.; Li, Y.; Li, F.; Zhou, X.; Wang, A.J. Sumoylation of turnip mosaic virus RNA polymerase promotes viral infection by counteracting the host NPR1-mediated immune response. Plant Cell 2017, 29, 508-525. [CrossRef]

13. Dufresne, P.J.; Thivierge, K.; Cotton, S.; Beauchemin, C.; Ide, C.; Ubalijoro, E.; Laliberté, J.F.; Fortin, M.G.J. Heat shock 70 protein interaction with Turnip mosaic virus RNA-dependent RNA polymerase within virus-induced membrane vesicles. Virology 2008, 374, 217-227. [CrossRef] [PubMed]

14. Huang, T.S.; Wei, T.; Laliberté, J.F.; Wang, A.J. A host RNA helicase-like protein, AtRH8, interacts with the potyviral genome-linked protein, $\mathrm{VPg}$, associates with the virus accumulation complex, and is essential for infection. Plant Physiol. 2010, 152, 255-266. [CrossRef] [PubMed] 
15. Li, Y.; Xiong, R.; Bernards, M.; Wang, A.J. Recruitment of Arabidopsis RNA helicase AtRH9 to the viral replication complex by viral replicase to promote turnip mosaic virus replication. Sci. Rep. 2016, 6, 30297. [CrossRef] [PubMed]

16. Andrés, M.S.A.; Arteaga, M.L.; Ortega, R.G.; Voorrips, R.E. New genes related to PVY resistance in C. annuum L. 'Serrano Criollo de Morelos-334'. In Proceedings of the Eucarpia Meeting on Genetics \& Breeding of Capsicum \& Eggplant, Noordwijkerhout, The Netherlands, 17-19 May 2004.

17. Cook, A.A.J. A mutation for resistance to Potato virus $Y$ in pepper. Phytopathology 1960, 51, 632.

18. Janzac, B.; Montarry, J.; Palloix, A.; Navaud, O.; Moury, B.J. A point mutation in the polymerase of Potato virus $Y$ confers virulence toward the Pvr4 resistance of pepper and a high competitiveness cost in susceptible cultivar. Mol. Plant Microbe Interact. 2010, 23, 823-830. [CrossRef]

19. Kyle, M.M.; Palloix, A.J. Proposed revision of nomenclature for potyvirusresistance genes in Capsicum. Euphytica 1997, 97, 183-188. [CrossRef]

20. Murphy, J.F.; Blauth, J.R.; Livingstone, K.D.; Lackney, V.K.; Jahn, M.K. Trichome density of main stem is tightly linked to PepMoV resistance in chili pepper (Capsicum annuum L.). Mol. Plant Microbe Interact. 2011, 122, 1051-1058.

21. Chen, M.; Sun, L.; Wu, H.; Chen, J.; Ma, Y.; Zhang, X.; Du, L.; Cheng, S.; Zhang, B.; Ye, X.J. Durable field resistance to Wheat yellow mosaic virus in transgenic wheat containing the antisense virus polymerase gene. Plant Biotechnol. J. 2014, 12, 447-456. [CrossRef]

22. Sun, L.; Andika, I.B.; Shen, J.; Yang, D.; Chen, J.J. The P2 of Wheat yellow mosaic virus rearranges the endoplasmic reticulum and recruits other viral proteins into replication-associated inclusion bodies. Mol. Plant Pathol. 2014, 15, 466-478. [CrossRef] [PubMed]

23. Singh, D.K.; Mcnellis, T.W.J. Fibrillin protein function: The tip of the iceberg? Trends Plant Sci. 2011, 16, 432-441. [CrossRef] [PubMed]

24. Tamura, K.; Dudley, J.; Nei, M.; Kumar, S. MEGA4: Molecular Evolutionary Genetics Analysis (MEGA) software version 4.0. Mol. Biol. Evol. 2007, 24, 1596-1599. [CrossRef] [PubMed]

25. Walter, M.; Chaban, C.; Schutze, K.O.; Weckermann, K.; Nake, C.; Blazevic, D.; Grefen, C.; Schumacher, K.; Oecking, C.J. Visualization of protein interactions in living plant cells using bimolecular fluorescence complementation. Plant J. 2010, 40, 428-438. [CrossRef]

26. Livak, K.J.; Schmittgen, T.D. Analysis of relative gene expression data using real-time quantitative PCR and the $2^{-\Delta \Delta C T}$ Method. Methods 2001, 25, 402-408. [CrossRef]

27. Cheng, Y.; Cui, L.; Lijie, Y.; Jackson, A.O.; Zhiyong, L.; Chenggui, H.; Jialin, Y.; Dawei, L.J. A high throughput barley stripe mosaic virus vector for virus induced gene silencing in monocots and dicots. PLOS ONE 2011,6, e26468.

28. Holzberg, S.; Brosio, P.; Gross, C.; Pogue, G.P. Barley stripe mosaic virus-induced gene silencing in a monocot plant. Plant J. 2002, 30, 315-327. [CrossRef]

29. Zhou, T.; Zhang, H.; Lai, T.; Cheng, Q.; Shi, N.; Wang, H.; Jin, M.; Zhong, S.; Fan, Z.; Liu, Y.J. Virus-induced gene complementation reveals a transcription factor network in modulation of tomato fruit ripening. Sci. Rep. 2012, 2, 836. [CrossRef]

30. Yael, L.D.; Marianna, O.; Elena, S.; Yigal, E.; Dalia, R.D.; Alexander, V.J. Expression and functional analyses of the plastid lipid-associated protein CHRC suggest its role in chromoplastogenesis and stress. Plant Physiol. 2006, 142, 233-244.

31. Laizet, Y.; Pontier, D.; Mache, R.; Kuntz, M.J. Subfamily organization and phylogenetic origin of genes encoding plastid lipid-associated proteins of the fibrillin type. J. Genome Sci. Technol. 2004, 3, 19-28. [CrossRef]

32. Bret, C.; Clarke, J.D.; Paul, B.; Joel, K.; Don, H.; Sylvia, P.; Sonia, G.; Molly, D.; Peter, L.; Cinzia, E.J. A network of rice genes associated with stress response and seed development. Proc. Natl. Acad. Sci. USA 2003, 100, 4945-4950.

33. Singh, D.K.; Maximova, S.N.; Jensen, P.J.; Lehman, B.L.; Ngugi, H.K.; Mcnellis, T.W. FIBRILLIN4 is required for plastoglobule development and stress resistance in apple and Arabidopsis. Plant Physiol. 2010, 154, 1281-1293. [CrossRef] [PubMed]

34. Vishnevetsky, M.; Ovadis, M.H.; Levy, M.; Libal, W.Y.; Adam, Z.; Vainstein, A.J. Molecular cloning of a carotenoid-associated protein from Cucumis sativus corollas: Homologous genes involved in carotenoid sequestration in chromoplasts. Plant J. 2010, 10, 1111-1118. [CrossRef] [PubMed] 
35. Yang, Y.; Sulpice, R.; Himmelbach, A.; Meinhard, M.; Christmann, A.; Grill, E.J. Fibrillin expression is regulated by abscisic acid response regulators and is involved in abscisic acid-mediated photoprotection. Proc. Natl. Acad. Sci. USA 2006, 103, 6061-6066. [CrossRef] [PubMed]

36. Alazem, M.; Lin, K.Y.; Lin, N.S.J. The abscisic acid pathway has multifaceted effects on the accumulation of Bamboo mosaic virus. Mol. Plant Microbe Interact. 2014, 27, 177-189. [CrossRef] [PubMed]

37. Fraser, R.S.S.; Whenham, R.J.J. Abscisic acid metabolism in tomato plants infected with tobacco mosaic virus: Relationships with growth, symptoms and the Tm-1 gene for TMV resistance. Physiol. Mol. Plant Pathol. 1989, 34, 215-226. [CrossRef]

38. Iriti, M.; Faoro, F.J. Abscisic acid is involved in chitosan-induced resistance to tobacco necrosis virus (TNV). Plant Physiol. Biochem. 2008, 46, 1106-1111. [CrossRef]

39. Deruère, J.; Römer, S.; d'Harlingue, A.; Backhaus, R.A.; Kuntz, M.; Camara, B. Fibril assembly and carotenoid overaccumulation in chromoplasts: A model for supramolecular lipoprotein structures. Plant Cell 1994, 6, 119-133.

40. Bai, Y.; Zhu, W.; Hu, X.; Sun, C.; Li, Y.; Wang, D.; Wang, Q.; Pei, G.; Zhang, Y.; Guo, A.J. Genome-wide analysis of the bZIP gene family identifies two ABI5-like bZIP transcription factors, BrABI5a and BrABI5b, as positive modulators of ABA signalling in Chinese cabbage. PLoS ONE 2016, 11, e0158966. [CrossRef]

41. Yang, T.; Yao, S.; Hao, L.; Zhao, Y.; Lu, W.; Xiao, K.J. Wheat bHLH-type transcription factor gene TabHLH1 is crucial in mediating osmotic stresses tolerance through modulating largely the ABA-associated pathway. Plant Cell Rep. 2016, 35, 2309-2323. [CrossRef]

(C) 2019 by the authors. Licensee MDPI, Basel, Switzerland. This article is an open access article distributed under the terms and conditions of the Creative Commons Attribution (CC BY) license (http://creativecommons.org/licenses/by/4.0/). 